\title{
Frugivoria por aves em um mosaico de Floresta Estacional Semidecidual e reflorestamento misto em Rio Claro, São Paulo, Brasil
}

\author{
Samira Athiê 1,2 $^{1,}$ Manoel Martins Dias ${ }^{1}$
}

Recebido em 28/05/2010. Aceito em 1/12/2011

\begin{abstract}
RESUMO
(Frugivoria por aves em um mosaico de Floresta Estacional Semidecidual e reflorestamento misto em Rio Claro, São Paulo, Brasil). Visando subsidiar futuros projetos de recuperação florestal com base nas interações animal- planta, foram avaliadas as espécies ornitocóricas e o consumo de frutos por aves em um mosaico de Floresta Estacional Semidecidual e um reflorestamento misto, em Rio Claro, São Paulo. Através do monitoramento da frutificação e avaliação dos eventos de frugivoria em sessões focais e ad libitum, foram registradas 31 espécies ornitocóricas fornecendo frutos para 38 espécies de aves consumidoras. No reflorestamento misto foram observadas $90,3 \%(n=28)$ das espécies ornitocóricas frutificando ao longo de todo ano, enquanto no fragmento florestal, somente $51,6 \%(n=16)$ delas foram registradas, com maiores variações temporais na oferta de frutos; $65,2 \%(n=1027)$ dos frutos foram consumidos no reflorestamento misto e $34,8 \%(n=547)$ no fragmento florestal. Tais resultados podem estar relacionados ao menor tamanho do fragmento de vegetação nativa, o que, proporcionalmente, poderia determinar uma menor riqueza de espécies vegetais no mesmo e/ou ao fato de a dispersão abiótica tender a predominar nos locais fragmentados e perturbados. O reflorestamento misto, embora apresente algumas espécies vegetais exóticas, está sendo ecologicamente mais funcional para a avifauna e, devido à proximidade com o fragmento, parece estar contribuindo para a manutenção das comunidades de aves residentes e visitantes deste último através dos recursos alimentares oferecidos.
\end{abstract}

Palavras-chave: consumo de frutos, interação ave-planta, Mata Atlântica

\begin{abstract}
(Frugivory by birds in a mosaic of seasonal semideciduous forest and a mixed reforested area in Rio Claro, Sao Paulo, Brazil). To support future forest recovery projects based on plant-animal interactions, this work aimed to evaluate the ornithochoric species and fruit consumption by birds in both a mosaic of seasonal semideciduous forest and a mixed reforested area in Rio Claro, Sao Paulo, Brazil. By monitoring and evaluating fruiting and frugivory events in focal and ad libitum sessions, 31 ornithochoric species were found that provide fruits to 38 species of frugivorous birds. In the mixed reforested area, $90.3 \%(n=28)$ of the ornithochoric species had fruit throughout the year, while in the forest fragment this percentage was only $51.6 \%(n=16)$, with higher temporal variations in the availability of the fruits; $65.2 \%(n=1027)$ of fruits were consumed in the reforested area and $34.8 \%(n=547)$ in the forest fragment. These results may be related to the smaller size of the forest fragment, which, proportionately, could determine a lower richness of plant species, and/or to the fact that abiotic dispersal tends to predominate in fragmented and disturbed sites. The mixed reforested area, although it has some exotic plant species, is ecologically more functional for birds and, due to its proximity to the fragment, apparently contributes to the maintenance of the resident and visiting bird communities of this region by providing food resources.
\end{abstract}

Key words: Atlantic Forest, bird-plant interaction, fruit consumption

1 Universidade Federal de São Carlos, Departamento de Ecologia e Biologia Evolutiva, São Carlos, SP, Brasil

2 Autor para correspondência: samira_esalq@yahoo.com.br 


\section{Introdução}

As Florestas Estacionais Semideciduais correspondem à formação vegetal mais rápida e extensamente devastada e fragmentada do estado de São Paulo, atualmente representadas, sobretudo, por pequenos fragmentos (Durigan et al. 2000). Considerando-se este quadro de desmatamento e impacto antrópico, os programas com objetivo de recuperar tais ecossistemas são de grande importância. Porém, para que esses projetos representem uma estratégia efetiva de conservação, é fundamental que se conheçam e se respeitem as interações ecológicas entre plantas e animais envolvidos, e se valer delas para estabelecer um processo contínuo de regeneração, possível de se auto-sustentar (Reis et al. 1999).

Mais recentemente, os programas de recuperação de áreas degradadas têm substituído a mera aplicação de práticas agronômicas ou silviculturais de plantios de espécies perenes por uma concepção de reconstrução de interações da comunidade, reconhecendo inclusive a importância das interações frugívoro-planta neste processo e recomendando a implantação de espécies nativas pioneiras e secundárias iniciais atrativas para a fauna (Rodrigues \& Gandolfi 2000). Frugívoros atraídos pelas espécies zoocóricas utilizadas no plantio não apenas dispersam as sementes dessas plantas, mas também trazem consigo sementes de outras espécies nativas, aumentando a riqueza específica da área (Silva 2003). Neste sentido, o processo de frugivoria contribui para a recolonização e reestruturação da vegetação através da dispersão de sementes (Wunderle 1997), e a avifauna, por sua vez, é beneficiada pela oferta de recursos alimentares, os quais possibilitam a manutenção e a fixação de suas populações nesses locais (Howe 1984). Sendo assim, é imprescindível garantir a disponibilidade de frutos atrativos para as aves em áreas de recomposição florestal ao longo de todo o ano, possibilitando que essa interação ave-planta seja mantida em curto e longo prazos (Reis et al. 1999).

O presente trabalho objetivou verificar a disponibilidade e o consumo de frutos por aves durante um ano em espécies vegetais ornitocóricas/potencialmente ornitocóricas em um mosaico de vegetação representado por um pequeno fragmento de Floresta Estacional Semidecidual e um reflorestamento misto com essências predominantemente nativas, localizados na região centro-leste do estado de São Paulo. Foi avaliada a funcionalidade ecológica dos ambientes através da interação ave-planta representada pelos processos de frugivoria e dispersão de sementes, projetos de recuperação florestal em áreas de Mata Atlântica, especialmente as Florestas Estacionais Semideciduais.

\section{Material e métodos}

\section{Área de estudo}

O estudo foi conduzido na Usina-Parque do Corumbataí (UPC), localizada na zona rural de Rio Claro, São Paulo
(22²9’S; 47³6’W). Segundo a classificação de Köeppen, o clima da região é do tipo Cwa, ou seja, tropical com duas estações bem definidas, sendo uma seca, de abril a setembro, e outra chuvosa, de outubro a março (Troppmair 1992). A área da UPC, que possui 44 ha, era originalmente coberta pela fitofisionomia de Floresta Estacional Semidecidual (domínio de Mata Atlântica), da qual permaneceram apenas 5 ha, constituindo o fragmento do presente estudo.

A vegetação desse fragmento apresenta uma estratificação simples, sendo possível distinguir três níveis principais: sub-bosque, dossel e árvores emergentes, estas dispostas esparsamente na área, tais como Chorisia speciosa St. Hill (Bombacaceae), Schizolobium parahyba (Vell.) Blake (Caesalpiniaceae) e Cariniana sp. (Lecythidacae). As lianas estão presentes em abundância em todos os estratos, chegando a encobrir muitas copas de árvores que se sobressaem do dossel ou que se localizam na borda. Por se tratar de um ecossistema bastante alterado, é possível encontrar em meio à vegetação algumas espécies exóticas, como Spathodea campanulata P. Beauv (Bignoniaceae), Psidium guajava L. (Myrtaceae) e Citrus sp. (Rutaceae). Adjacente ao fragmento encontra-se uma área de reflorestamento misto, o qual foi implantado em duas fases (1982 e 2001), ocupando um total de 27 ha. O projeto inicialmente contemplou 70 espécies vegetais (CESP 1982), principalmente de Mata Atlântica, além de algumas frutíferas exóticas, tais como Artocarpus integrifolia L. (Moraceae), Melia azedarach L. (Meliaceae) e Syzygium jambolana (Lam.) DC. (Myrtaceae).

\section{Metodologia}

Através de inspeções quinzenais nas bordas, em trilhas pré-existentes e em dois transectos abertos no reflorestamento, com cerca de $200 \mathrm{~m}$ cada, e uma trilha de aproximadamente $100 \mathrm{~m}$ no fragmento florestal, entre abril de 2008 e março de 2009 , foram selecionadas as espécies vegetais que pudessem ser incluídas na síndrome de ornitocoria (endozoocoria) descrita por Pijl (1969). As espécies que apresentaram pelo menos um indivíduo com relativa abundância de frutos e ao menos um registro de frugivoria ad libitum, foram avaliadas através de observações focais em sessões de 1 hora, totalizando entre 4 e 30 horas para cada espécie. O tempo de observação variou entre as espécies principalmente em função da disponibilidade de frutos maduros. As coletas de dados foram realizadas entre 7:00 h e 13:00 h, com auxílio de binóculo 10 x 25, a uma distância mínima de $10 \mathrm{~m}$ da planta.

Foram realizadas as seguintes mensurações nas espécies estudadas sistematicamente: dimensões dos frutos (comprimento e diâmetro), massa dos frutos e sementes, número de sementes/fruto e proporção de polpa $\left(\mathrm{P}_{\mathrm{p}}\right)$. Para cada espécie foi utilizada amostra de 30 frutos maduros. As dimensões foram obtidas com auxílio de paquímetro (precisão de $0,1 \mathrm{~mm}$ ) e as massas utilizando-se balança digital (precisão de $0,1 \mathrm{~g}$ ). A $\mathrm{P}_{\mathrm{p}}$ foi obtida subtraindo-se a massa do 
lote de sementes contidas nos 30 frutos da massa total dos frutos, dividindo-se o valor obtido pela massa total destes e multiplicando-se o resultado por 100 (Argel-de-Oliveira 1999). Com a finalidade de verificar a similaridade na riqueza de espécies vegetais ornitocóricas entre os ambientes estudados (reflorestamento e fragmento), foi calculado o Índice de Similaridade de Jaccard (ISJ), dado por: $\mathrm{ISJ}=[\mathrm{C} /$ $(\mathrm{R}+\mathrm{F}+\mathrm{C})]^{\star} 100$, onde: $\mathrm{R}=$ número de espécies exclusivas do reflorestamento misto; $\mathrm{F}=$ número de espécies exclusivas do fragmento florestal; $\mathrm{C}=$ número de espécies comuns às duas áreas.

Durante as sessões de observação dos eventos de frugivoria, os seguintes dados foram registrados: espécies de aves visitantes, número de frutos consumidos, tempo de permanência sobre a planta e comportamentos de consumo dos frutos. Quanto ao modo de consumo, as aves foram agrupadas em três categorias: a) aquelas que engoliram os frutos inteiros, atuando como potenciais dispersoras, b) as que consumiram pedaços da polpa e c) aquelas que mandibularam os frutos, consideradas potenciais predadoras das sementes (Pizo 1997).

A porcentagem relativa de consumo (PC) foi obtida dividindo-se o número total de frutos consumidos por espécie pela soma dos frutos consumidos por todas as espécies, e multiplicando-se o valor obtido por 100. Apenas as visitas completas (quando a ave pôde ser acompanhada desde sua chegada até a saída) foram utilizadas para calcular as médias do tempo de duração das visitas e do número de frutos consumidos por visita (Krügel et al. 2006). Dados sobre a dieta e status de permanência na área seguem Willis (1979) e Sick (1997). A nomenclatura e a ordem taxonômica das espécies seguem os padrões do Comitê Brasileiro de Registros Ornitológicos (CBRO 2009).

\section{Resultados}

\section{Espécies vegetais}

Foram identificadas 31 espécies potencialmente ornitocóricas, distribuídas em 16 famílias, frutificando ao longo do período de estudo, das quais 22 apresentaram registros de consumo (Tab.1). No reflorestamento misto foram observadas $90,3 \%(n=28)$ dessas espécies, enquanto no fragmento florestal, somente $51,6 \%(\mathrm{n}=16)$ delas foram registradas, obtendo-se uma similaridade (ISJ) de 41,2\% entre esses ambientes em relação à composição de espécies ornitocóricas.

Durante a maior parte da época seca (abril a julho) foi registrada apenas uma espécie ornitocórica frutificando no fragmento de Floresta Estacional Semidecidual: Urera baccifera, que apresentou frutos maduros somente no mês de junho (Tab. 1). Já no reflorestamento misto foram observadas três espécies com frutos maduros em tal período: S. terebinthifolius, M. azedarach e U. baccifera (Tab. 1). A espécie nativa S. terebinthifolius, conhecida por apresentar frutos bastante atrativos à avifauna (Lorenzi 1992) foi a menos visitada $(0,05 \pm 0$ visitas/h), enquanto a espécie exótica $M$. azedarach recebeu a maior proporção de visitas alimentares $(7 \pm 3,7 / \mathrm{h})$.

Em 242 horas totais de observações focais foram consumidos 1574 frutos durante 801 visitas alimentares (Tab.2), em uma média de 3,3 $\pm 3,8$ visitas alimentares/h. Deste total, $65,2 \%(n=1027)$ dos frutos foram consumidos no reflorestamento misto e $34,8 \%(n=547)$ no fragmento florestal. Ocorreram, respectivamente, 3,0 visitas alimentares por hora no reflorestamento misto e 2,7 no fragmento florestal. As maiores taxas médias de visitação ocorreram em espécies com frutos dos tipos cápsula $(5,5$ visitas/h) e baga ( 3,5 visitas/h), enquanto o consumo foi proporcionalmente mais elevado em plantas com frutos dos tipos cápsula ( 8,5 frutos/h) e drupa ( 6,5 frutos/h), como mostra a Figura 1.

Dentre as espécies acompanhadas sistematicamente, $61,5 \%$ apresentaram frutos pequenos (diâmetro $\leq 1,0 \mathrm{~cm}$ ) e $38,5 \%$ frutos médios (diâmetro $>1,0-2,0 \mathrm{~cm}$ ), predominando os frutos carnosos indeiscentes e a coloração vermelha (Tab.3). As espécies que apresentaram maior proporção de polpa em seus frutos foram, em ordem decrescente, Acnistus arborescens, Ficus citrifolia e Myrciaria cauliflora, enquanto Schinus terebinthifolius apresentou a menor proporção de polpa dentre as espécies estudadas. O número de sementes por fruto variou de uma unidade, em S. terebinthifolius, Syagrus romanzoffiana, Eugenia jambolana, Rhamnus purshiana e Trema micrantha, até uma média de 4464 sementes nas infrutescências de Cecropia sp., no entanto predominaram espécies com frutos contendo em média até 10 sementes $(69,2 \%, n=9)$, como pode ser observado na Tabela 3 .

Mais da metade dos frutos consumidos foram ingeridos inteiros, sem danos às sementes, tendo sido de $51,1 \%$ a potencial proporção de sementes dispersas na área de estudo. Já o potencial de predação foi de, no máximo, $15,0 \%$, considerando-se a taxa de frutos mandibulados pelas aves antes da ingestão e, levando-se em conta que, nem todos os frutos mandibulados têm as sementes inviabilizadas. A espécie que apresentou a maior taxa de visitação e de frutos consumidos foi Melia azedarach (Tab.2), a qual também apresentou a maior taxa de frutos consumidos aos pedaços, permanecendo as sementes nos restos dos frutos na própria planta (Tab.3). Casearia silvestris apresentou o maior potencial de sementes dispersas, já que a grande maioria dos frutos não foi mandibulada (excetuando-se S. terebinthifolius, cujas remoções resultaram em $100 \%$ de frutos engolidos sem mandibulação, porém apenas uma visita alimentar foi registrada para a espécie durante as sessões). A espécie Cecropia sp. apresentou a maior taxa de frutos mandibulados (100\%); no entanto é possível inferir que ainda assim apresenta grande potencial de dispersão, pois em cada uma de suas infrutescências há geralmente mais de 4 mil minúsculas sementes (Tab. 3). 
Tabela 1. Plantas ornitocóricas/potencialmente ornitocóricas registradas frutificando no mosaico vegetacional, em Rio Claro, São Paulo, entre abril de 2008 e março de 2009.

\begin{tabular}{|c|c|c|c|c|}
\hline Família/Espécie & Nome popular & Porte & Ocorrência $^{1}$ & Meses \\
\hline \multicolumn{5}{|l|}{ Anacardiaceae } \\
\hline Schinus terebinthifolius Raddi $^{*}$ & aroeira-pimenteira & arvoreta & re & out/ago \\
\hline \multicolumn{5}{|l|}{ Arecaceae } \\
\hline Syagrus oleracea (Mart.) Becc." & guariroba & palmeira & re & nov \\
\hline Syagrus romanzoffiana (Cham.) Glass.. & jerivá & palmeira & re & ago/set \\
\hline \multicolumn{5}{|l|}{ Cactaceae } \\
\hline Pereskia aculeata Mill. & ora-pro-nóbis & arbusto & $\mathrm{ma}$ & ago/set \\
\hline \multicolumn{5}{|l|}{ Euphorbiaceae } \\
\hline Alchornea sidifolia Müll. Arg.* & tapiá & arvoreta & $\mathrm{ma}$ & out $/$ nov \\
\hline \multicolumn{5}{|l|}{ Lacistemaceae } \\
\hline Lacistema aggregatum (Berg.) Rusby & mata-calado & arvoreta & re & out/nov \\
\hline \multicolumn{5}{|l|}{ Malvaceae } \\
\hline Guazuma ulmifolia Lam.* & mutamba & árvore & re & mar \\
\hline \multicolumn{5}{|l|}{ Meliaceae } \\
\hline Melia azedarach L. & cinamomo & árvore & re & $\mathrm{abr} / \mathrm{dez}$ \\
\hline Trichilia claussenii C. DC.* & catiguá & árvore & re & $\mathrm{dez} / \mathrm{fev}$ \\
\hline \multicolumn{5}{|l|}{ Moraceae } \\
\hline Ficus citrifolia Mill. * & figueira & árvore & re & out/dez \\
\hline Ficus guaranitica Chodat \& Vischer ${ }^{*}$ & figueira & árvore & $\mathrm{re}, \mathrm{ma}$ & set/out/nov \\
\hline Maclura tinctoria (L.) D. Don. Steud.* & taiúva & árvore & re,ma & out/nov \\
\hline \multicolumn{5}{|l|}{ Myrtaceae } \\
\hline Campomanesia sp. & - & arvoreta & re & fev \\
\hline Eugenia jambolana Lam.* & jambolão & árvore & re & $\mathrm{jan} / \mathrm{fev}$ \\
\hline Eugenia uniflora L.* & pitangueira & arvoreta & re & nov \\
\hline Myrciaria cauliflora Mart. (O.) Berg.* & jabuticabeira & árvore & re & out/nov \\
\hline Psidium guajava $\mathrm{L} . *$ & goiabeira & arvoreta & re, ma & jan,fev \\
\hline Psidium sp. & - & arvoreta & re & nov, dez \\
\hline \multicolumn{5}{|l|}{ Piperaceae } \\
\hline Piper sp. $1^{*}$ & - & arbusto & re, ma & set/out/nov/dez \\
\hline Piper sp. 2 & - & arbusto & re, ma & set/out/nov/dez \\
\hline \multicolumn{5}{|l|}{ Rhamnaceae } \\
\hline Rhamnus purshiana DC.* & cáscara-sagrada & arvoreta & re, ma & $\mathrm{dez} / \mathrm{jan}$ \\
\hline \multicolumn{5}{|l|}{ Salicaceae } \\
\hline Casearia sylvestris Sw.. & guaçatonga & arvoreta & re, ma & set/out \\
\hline \multicolumn{5}{|l|}{ Solanaceae } \\
\hline Acnistus arborescens (L.) Schltdl.* & fruta-de-sabiá & arbusto & re, ma & out/dez \\
\hline Solanum granulosoleprosum Dunal ${ }^{*}$ & jurubeba & arvoreta & re, ma & ago/dez \\
\hline Solanum nigrum $\mathrm{L}$. & maria-preta & erva & re, ma & out/nov \\
\hline \multicolumn{5}{|l|}{ Ulmaceae } \\
\hline Celtis sp. & grão-de-galo & liana & re & nov \\
\hline Trema micrantha (L.) Blume ${ }^{+}$ & pau-pólvora & arvoreta & $\mathrm{ma}$ & $\mathrm{fev} / \mathrm{mar}$ \\
\hline \multicolumn{5}{|l|}{ Urticaceae } \\
\hline Cecropia pachystachya Trec. & embaúba-branca & árvore & re, ma & set/out \\
\hline Cecropia spp.* & embaúba & árvore & re & $\mathrm{ago} / \mathrm{fev}$ \\
\hline Urera baccifera (L.) Gaudich." & urtiga & arbusto & re, ma & jun \\
\hline \multicolumn{5}{|l|}{ Verbenaceae } \\
\hline Lantana camara $\mathrm{L}$. & lantana & erva & $\mathrm{re}, \mathrm{ma}$ & nov \\
\hline
\end{tabular}

${ }^{1}$ re=reflorestamento misto, ma=fragmento de mata nativa. "espécies em que houve pelo menos um registro de consumo (ad libitum ou sistemático). 


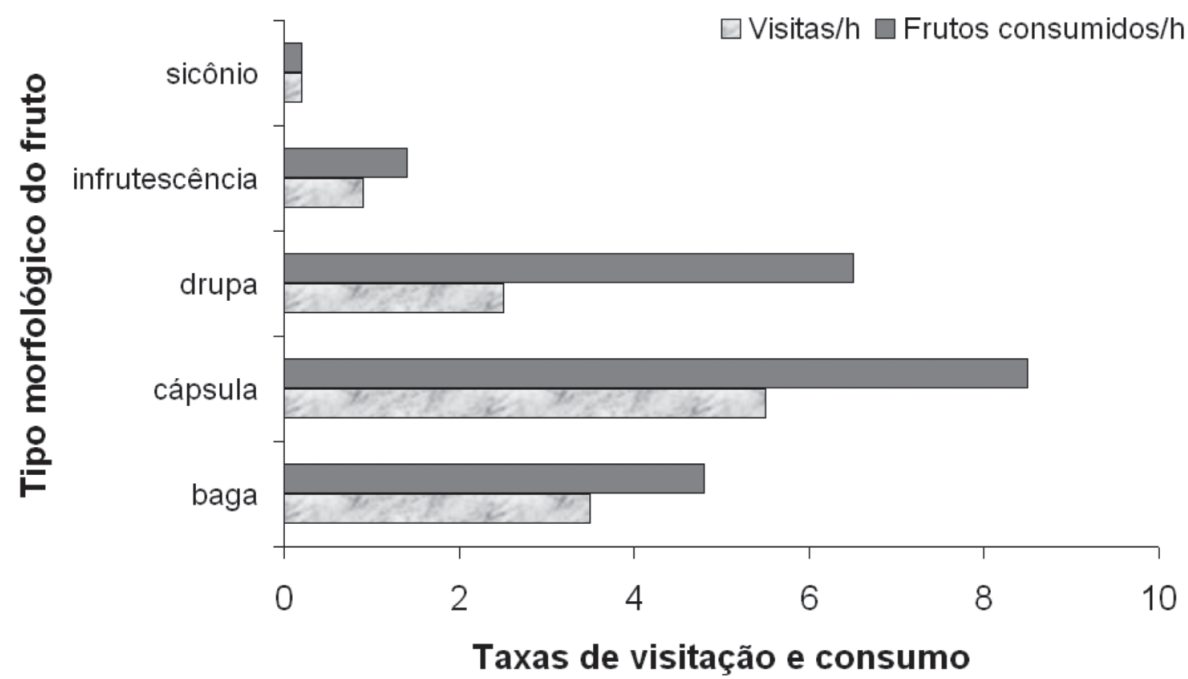

Figura 1. Taxas médias de visitação e consumo (por hora), em função do tipo morfológico do fruto no mosaico vegetacional em Rio Claro, São Paulo.

Tabela 2. Lista das plantas ornitocóricas acompanhadas sistematicamente no mosaico vegetacional em Rio Claro, São Paulo, seguida das informações sobre disponibilidade temporal dos frutos, tempo de observação e detalhes de visitação e consumo.

\begin{tabular}{|c|c|c|c|c|c|c|c|c|c|c|c|c|c|c|c|c|c|c|}
\hline \multirow{2}{*}{$\begin{array}{l}\text { Família/Espécie } \\
\text { vegetal }\end{array}$} & \multicolumn{11}{|c|}{$\begin{array}{l}\text { Disponibilidade de } \\
\text { frutos maduros }\end{array}$} & \multirow{2}{*}{$\begin{array}{c}\text { Tempo de } \\
\text { observação } \\
(\mathrm{h})\end{array}$} & \multirow{2}{*}{$\begin{array}{c}\mathrm{N}^{\circ} \text { de espécies } \\
\text { de aves } \\
\text { consumidoras }\end{array}$} & \multirow{2}{*}{$\begin{array}{c}\mathrm{N}^{\circ} \text { total } \\
\text { de visitas } \\
\text { alimentares }^{2}\end{array}$} & \multirow{2}{*}{$\begin{array}{c}\mathrm{N}^{\circ} \text { total } \\
\text { de frutos } \\
\text { consumidos }\end{array}$} & \multirow{2}{*}{$\begin{array}{c}\text { Duração } \\
\text { das } \\
\text { visitas }^{2,3}\end{array}$} & \multirow{2}{*}{$\begin{array}{l}\text { Consumo/ } \\
\text { visita }^{2,4}\end{array}$} & \multirow{2}{*}{$\begin{array}{l}\text { Taxa de } \\
\text { consumo }\end{array}$} \\
\hline & $\mathrm{J}$ & $\mathrm{F}$ & $\mathrm{M}$ & $\mathrm{A}$ & $\mathrm{M}$ & $\mathrm{J} \mathrm{J}$ & $\mathrm{A}$ & $\mathrm{S}$ & $\mathrm{O}$ & $\mathrm{N}$ & $\mathrm{D}$ & & & & & & & \\
\hline \multicolumn{19}{|l|}{ Anacardiaceae } \\
\hline $\begin{array}{l}\text { Schinus } \\
\text { terebinthifolius }\end{array}$ & $\mathrm{x}$ & $\mathrm{x}$ & $\mathrm{x}$ & $\mathrm{x}$ & $\mathrm{x}$ & $x^{x}$ & $\mathrm{x}$ & & $\mathrm{x}$ & $\mathrm{x}$ & $\mathrm{x}$ & 20 & 8 & 1 & 2 & $120 \pm 0$ & $2 \pm 0$ & $0,1 \pm 0$ \\
\hline \multicolumn{19}{|l|}{ Arecaceae } \\
\hline $\begin{array}{l}\text { Syagrus } \\
\text { romanzoffiana }\end{array}$ & & & & & & & $\mathrm{x}$ & $\mathrm{x}$ & & & & 4 & 2 & 3 & 7 & $5 \pm 0$ & $1 \pm 0$ & $1,75 \pm 3,5$ \\
\hline \multicolumn{19}{|l|}{ Meliaceae } \\
\hline Melia azedarach & & & & $\mathrm{x}$ & $\mathrm{x}$ & $\mathrm{x} \quad \mathrm{x}$ & $\mathrm{x}$ & $\mathrm{x}$ & $\mathrm{x}$ & $\mathrm{x}$ & $\mathrm{x}$ & 30 & 20 & 210 & 414 & $181 \pm 163$ & $2,5 \pm 1,4$ & $13,8 \pm 8,4$ \\
\hline $\begin{array}{l}\text { Trichilia } \\
\text { claussenii }\end{array}$ & $\mathrm{x}$ & $\mathrm{x}$ & & & & & & & & & $\mathrm{x}$ & 30 & 18 & 169 & 224 & $110,8 \pm 68,2$ & $2,0 \pm 0,9$ & $7,5 \pm 5,2$ \\
\hline \multicolumn{19}{|l|}{ Moraceae } \\
\hline Fícus citrifolia & & & & & & & & & $\mathrm{x}$ & $\mathrm{x}$ & $\mathrm{x}$ & 10 & 4 & 2 & 2 & $50,0 \pm 0$ & $1,0 \pm 0$ & $0,2 \pm 0,4$ \\
\hline \multicolumn{19}{|l|}{ Myrtaceae } \\
\hline $\begin{array}{l}\text { Eugenia } \\
\text { jambolana }\end{array}$ & $\mathrm{x}$ & $\mathrm{x}$ & & & & & & & & & & 10 & 8 & 27 & 37 & $56,8 \pm 41,8$ & $1,2 \pm 0,5$ & $3,7 \pm 3,6$ \\
\hline $\begin{array}{l}\text { Myrciaria } \\
\text { cauliflora }\end{array}$ & & & & & & & & & $\mathrm{x}$ & $\mathrm{x}$ & & 12 & 11 & 82 & 106 & $88,4 \pm 41,2$ & $1,4 \pm 0,5$ & $8,8 \pm 7,6$ \\
\hline \multicolumn{19}{|l|}{ Rhamnaceae } \\
\hline $\begin{array}{l}\text { Rhamnus } \\
\text { purshiana }\end{array}$ & $\mathrm{x}$ & & & & & & & & & & $\mathrm{x}$ & 23 & 4 & 67 & 163 & $55,0 \pm 16,1$ & $3,0 \pm 1,5$ & $7,1 \pm 5,3$ \\
\hline \multicolumn{19}{|l|}{ Salicaceae } \\
\hline $\begin{array}{l}\text { Casearia } \\
\text { sylvestris }\end{array}$ & & & & & & & & $\mathrm{x}$ & $\mathrm{x}$ & & & 22 & 18 & 118 & 206 & $104,8 \pm 66,7$ & $1,7 \pm 0,9$ & $9,4 \pm 8$ \\
\hline \multicolumn{19}{|l|}{ Solanaceae } \\
\hline $\begin{array}{l}\text { Acnistus } \\
\text { arborescens }\end{array}$ & & & & & & & & & $\mathrm{x}$ & $\mathrm{x}$ & $\mathrm{x}$ & 18 & 15 & 64 & 96 & $43,0 \pm 36,2$ & $2,1 \pm 1,0$ & $5,4 \pm 3,8$ \\
\hline $\begin{array}{l}\text { Solanum } \\
\text { granulosoleprosum }\end{array}$ & & & & & & & $\mathrm{x}$ & $\mathrm{x}$ & $\mathrm{x}$ & $\mathrm{x}$ & $\mathrm{x}$ & 23 & 3 & 4 & 6 & - & - & $0,2 \pm 1$ \\
\hline \multicolumn{19}{|l|}{ Ulmaceae } \\
\hline $\begin{array}{l}\text { Trema } \\
\text { micrantha }\end{array}$ & & $\mathrm{x}$ & $\mathrm{x}$ & & & & & & & & & 23 & 6 & 39 & 288 & $77,0 \pm 73,0$ & $4,0 \pm 1,6$ & $12,5 \pm 21,7$ \\
\hline \multicolumn{19}{|l|}{ Urticacae } \\
\hline Cecropia sp. & $\mathrm{x}$ & $\mathrm{x}$ & & & & & $\mathrm{x}$ & $\mathrm{x}$ & $\mathrm{x}$ & $\mathrm{x}$ & $\mathrm{x}$ & 17 & 6 & 15 & 23 & $57,7 \pm 45$ & $1,2 \pm 0,4$ & $1,4 \pm 2,0$ \\
\hline Total & & & & & & & & & & & & 242 & - & 801 & 1574 & $132,1 \pm 135,4$ & $2,3 \pm 1,4$ & $6,5 \pm 9,5$ \\
\hline
\end{tabular}

${ }^{1}$ Foram incluídas as espécies registradas ad libitum; ${ }^{2}$ refere-se apenas às sessões focais; ${ }^{3}$ média do tempo de duração de cada visita (em segundos) \pm desvio padrão; ${ }^{4}$ média do número de frutos consumidos por visita \pm desvio padrão; ${ }^{5}$ número de frutos consumidos por hora para cada espécie \pm desvio padrão. 
Tabela 3. Características qualitativas e quantitativas de frutos e sementes das espécies estudadas sistematicamente no mosaico vegetacional em Rio Claro, São Paulo, e modos de consumo dos frutos.

\begin{tabular}{|c|c|c|c|c|c|c|c|c|c|c|c|}
\hline \multirow[b]{2}{*}{$\begin{array}{l}\text { Família/Espécie } \\
\text { vegetal }\end{array}$} & \multicolumn{5}{|c|}{ Frutos } & \multicolumn{2}{|c|}{ Sementes } & \multirow[b]{2}{*}{$\mathrm{P}_{\mathrm{p}}{ }^{2}$} & \multicolumn{3}{|c|}{ Consumo $^{3}$} \\
\hline & Tipo & Cor & $\begin{array}{l}\text { Comprimento } \\
\text { médio }(\mathrm{cm})\end{array}$ & $\begin{array}{l}\text { Diâmetro } \\
\text { médio } \\
(\mathrm{cm})\end{array}$ & $\begin{array}{l}\text { Massa } \\
\text { média } \\
(\mathrm{g})\end{array}$ & $\begin{array}{l}\text { Massa }^{1} \\
\text { média } \\
(\mathrm{g})\end{array}$ & $\begin{array}{l}\mathrm{N}^{\mathrm{O}} \\
\text { médio/ } \\
\text { fruto }\end{array}$ & & $\begin{array}{l}\text { Frutos } \\
\text { engolidos }\end{array}$ & $\begin{array}{c}\text { Frutos } \\
\text { consumidos } \\
\text { aos pedaços }\end{array}$ & $\begin{array}{c}\text { Frutos } \\
\text { mandibulados }\end{array}$ \\
\hline $\begin{array}{l}\text { Schinus } \\
\text { terebinthifolius }\end{array}$ & drupa & vermelha & 0,4 & 0,5 & $1,6 \times 10^{-2}$ & $1,3 \times 10^{-2}$ & 1,0 & 20,0 & $2(100)$ & 0 & 0 \\
\hline $\begin{array}{l}\text { Syagrus } \\
\text { romanzoffiana }{ }^{\mathrm{a}}\end{array}$ & drupa & amarela & 2,5 & 2,0 & 9,4 & 4,7 & 1,0 & 50,0 & 0 & $7(100)$ & 0 \\
\hline Melia azedarach & drupa & amarela & 1,3 & 1,2 & 1,2 & $0,7^{\mathrm{b}}$ & 4,0 & 44,2 & $96(23,2)$ & $316(76,3)$ & $2(0,5)$ \\
\hline $\begin{array}{l}\text { Trichilia } \\
\text { claussenii }\end{array}$ & cápsula & vermelha & 9,6 & 9,4 & 0,5 & 0,1 & 1,4 & 73,9 & $128(57,1)$ & $80(35,7)$ & $16(7,2)$ \\
\hline Fícus citrifolia & sicônio & verde/rósea & 10,2 & 9,0 & 0,4 & $1,36 \times 10^{-4}$ & 296,4 & 90,9 & $1(50,0)$ & $1(50,0)$ & 0 \\
\hline $\begin{array}{l}\text { Eugenia } \\
\text { jambolana }\end{array}$ & drupa & $\begin{array}{c}\text { atro- } \\
\text { purpúrea }\end{array}$ & 20,5 & 14,3 & 2,6 & 0,3 & 1,0 & 86,7 & $4(10,8)$ & $32(86,5)$ & $1(2,7)$ \\
\hline $\begin{array}{l}\text { Myrciaria } \\
\text { cauliflora }\end{array}$ & baga & preta & 18,0 & 17,7 & 3,5 & 0,2 & 2,7 & 89,4 & 0 & $79(79,8)$ & $20(20,2)$ \\
\hline $\begin{array}{l}\text { Rhamnus } \\
\text { purshiana }\end{array}$ & drupa & vermelha & 10,6 & 9,0 & 0,6 & 0,1 & 1,0 & 75,3 & $140(86,4)$ & $1(0,6)$ & $21(13,0)$ \\
\hline $\begin{array}{l}\text { Casearia } \\
\text { sylvestris }\end{array}$ & cápsula & vermelha & 0,4 & 0,4 & $2 \times 10^{-2}$ & $1,6 \times 10^{-2}$ & 4,8 & 83,3 & $180(87,4)$ & 0 & $26(12,6)$ \\
\hline $\begin{array}{l}\text { Acnistus } \\
\text { arborescens }\end{array}$ & baga & laranja & 6,05 & 6,27 & 0,15 & $8,5 \times 10^{-2}$ & 12,7 & 95,9 & $21(21,9)$ & $9(9,4)$ & $66(68,7)$ \\
\hline $\begin{array}{l}\text { Solanum } \\
\text { granulosoleprosum }\end{array}$ & baga & amarela & 1,4 & 1,3 & 1,5 & 0,2 & 132,1 & 83,8 & 0 & $5(100)$ & 0 \\
\hline Trema micrantha & drupa & vermelha & 3,5 & 3,0 & $1,7 \times 10^{-2}$ & $6,7 \times 10^{-3}$ & 1,0 & 60 & $228(79,2)$ & 0 & $60(20,8)$ \\
\hline Cecropia sp. & Infrutescência & verde & 12,8 & 1,0 & 8,6 & 4,4 & $4464,3^{c}$ & 51,3 & 0 & 0 & $23(100)$ \\
\hline & & & Total & & & & & & $800(51,1 \%)$ & $530(33,9 \%)$ & $235(15,0 \%)$ \\
\hline
\end{tabular}

${ }^{1}$ Média da massa de sementes contidas em cada fruto; ${ }^{2}$ proporção de polpa; ${ }^{3}$ porcentagem entre parênteses; aextraído de CASTRO \& GALETTI (2004); ${ }^{\text {bmédia da }}$ massa das cápsulas lenhosas que contêm as sementes, somada à massa destas; 'excepcionalmente para essa característica utilizou-se amostra de três infrutescências.

\section{Avifauna consumidora}

Foram registradas 38 espécies de aves, pertencentes a 11 famílias, consumindo frutos nos ecossistemas estudados. Das espécies observadas forrageando durante as sessões focais ( $\mathrm{n}=30$, excluindo-se Elaenia sp.), $74,2 \%$ são onívoras, $19,4 \%$ insetívoras e 6,4\% frugívoras, as quais foram responsáveis respectivamente por 96,6\%, 3,3\% e $0,1 \%$ do consumo de frutos,. As famílias mais representativas foram Tyrannidae (11), responsável por 11,8\% do consumo, e Thraupidae (9), cujos representantes consumiram 70,5\% dos frutos (Fig.2). Thraupis sayaca foi responsável pela maior porcentagem relativa de consumo $(29,0 \%)$, seguida por Dacnis cayana, que consumiu $15,8 \%$ dos frutos (Tab.4). Os traupídeos T. sayaca, Ramphocelus carbo e Tangara cayana, demonstraram um maior grau de interação, consumindo frutos de, respectivamente, $63,6 \%(n=14), 50,0 \%(n=11)$ e 50,0\% ( $n=11)$, das espécies identificadas como ornitocóricas em que houve registros de consumo.

Quanto à manipulação dos frutos, $51,1 \%$ foram engolidos inteiros, $33,9 \%$ consumidos aos pedaços e $15,0 \%$ mandibulados. Dentre as espécies que engoliram os frutos inteiros, atuando como potenciais dispersoras destacam-se as pertencentes às famílias Turdidae $\mathrm{e}$ Tyrannidae.

\section{Discussão}

\section{Espécies vegetais}

Nos trópicos, a maior parte das espécies arbóreas com frutos atrativos à avifauna frutifica na época de chuvas, entre outubro e março (Foster 1977; Fleming et al. 1987), como constatado no presente estudo. Desta forma, espécies que frutificam na época seca (entre abril e setembro), são de suma importância no que diz respeito ao suprimento alimentar de aves que incluem frutos em sua dieta (Foster 1977). Durante a estação seca, quando há também menor abundância de insetos nos trópicos, a disponibilidade de frutos passa a ser crucial inclusive para as aves que os consomem como recurso alternativo ou complementar (Howe \& Estabrook 1977). Porém, os resultados do presente estudo mostraram que há uma maior riqueza de espécies vegetais atrativas à avifauna no reflorestamento misto, em detrimento do fragmento florestal, e que a oferta de frutos é muito mais variável ao longo do ano neste último. Tais fatos podem estar relacionados a dois fatores: a) o menor tamanho do fragmento de vegetação nativa, o que poderia determinar uma menor riqueza proporcional de espécies vegetais no mesmo; b) em locais fragmentados e mais perturbados, a dispersão abiótica tende a predominar, devido à redução da riqueza e do tamanho das populações de animais dispersores, de acordo com Morellato (1991). 


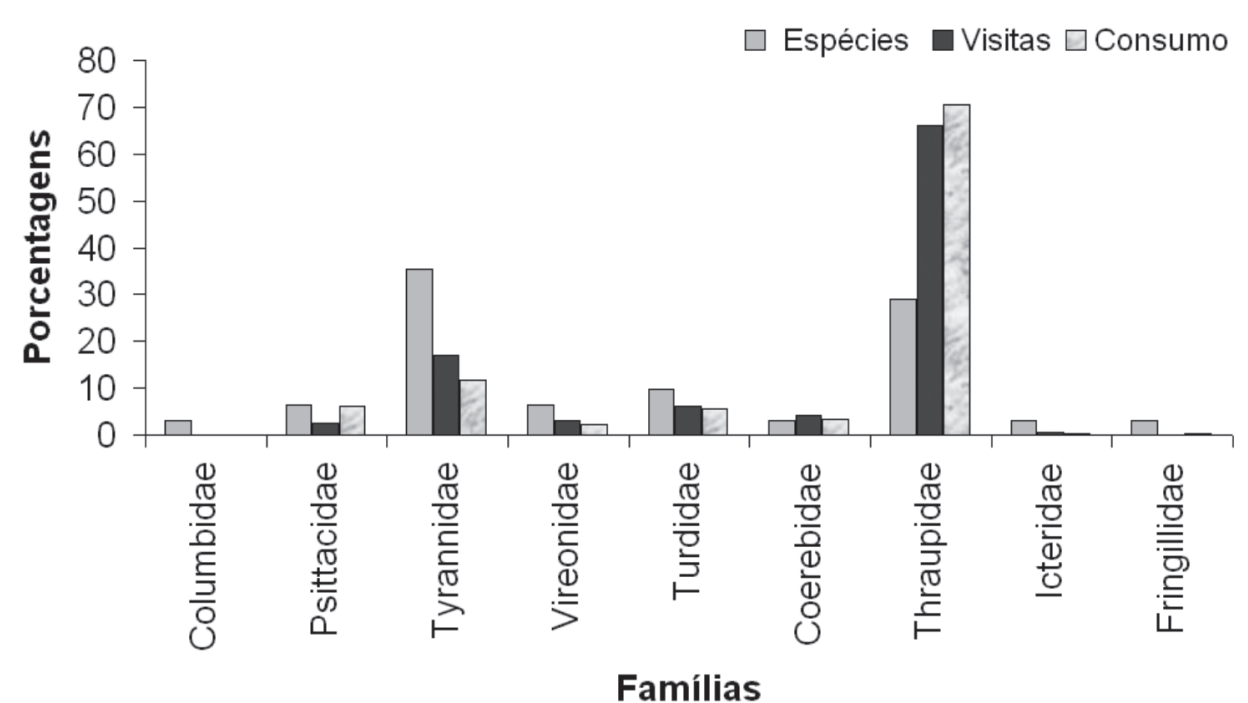

Figura 2. Representatividade de cada família de aves em relação à proporção de espécies, visitas e consumo de frutos, no mosaico vegetacional em Rio Claro, São Paulo.

Os resultados mostraram uma preferência da avifauna local pelos frutos vermelhos. O maior consumo de frutos com colorações chamativas é freqüente em espécies de plantas generalistas, que se valem dessa conspicuidade para atrair dispersores oportunistas em abundância (Molinari 1993). A preferência das aves por tal padrão de cor parece ser universal, tendo sido registrada em diversos estudos realizados em florestas tropicais e temperadas. Este padrão pode estar relacionado com a maior sensibilidade de alguns grupos de aves à região de ondas longas do espectro de luz visível (Stiles 1976).

A reduzida taxa de consumo em $S$. romanzoffiana se deve à baixa freqüência de aves frugívoras de grande porte especializadas no consumo de diásporos de tamanhos maiores, fato comum em matas pequenas e alteradas (Jordano et al. 2006). No caso dos frutos de S. granulosoleprosum, estes são consumidos principalmente por mamíferos, segundo Cáceres \& Moura (2003), que registraram sete espécies de mamíferos e apenas duas de aves consumindo frutos desta espécie em área de vegetação perturbada em Curitiba, Paraná. Enquanto em S. terebinthifolius, o baixo consumo pode ter sido decorrente de dois outros fatores: a) uma diluição dos eventos de frugivoria em muitas plantas da espécie, a qual é abundante na área de estudo, mascarando seu potencial de atração através da metodologia utilizada - hipótese reforçada pelo maior número de diagnósticos de consumo realizados ad libitum (Tab. 4) e/ou b) por uma possível preferência alimentar das aves pelos frutos de $M$. azedarach - hipótese reforçada pela maior proporção de polpa em seus frutos (Tab. 3) e por ser a única espécie que frutificou durante todo o outono, além de S. terebinthifolius. Conseqüentemente, a elevada taxa de frugivoria em M. azedarach, pode ser derivada da hipótese "b". Segundo Gosper et al. (2005), a frutificação em épocas de escassez de alimentos e a maior atração dos frutos em detrimento de espécies nativas são importantes estratégias de plantas exóticas invasoras, que muitas vezes se valem da reduzida concorrência e da preferência alimentar dos agentes dispersores para se propagarem e colonizarem novos sítios.

A elevada proporção de frutos engolidos inteiros e a significativa taxa de consumo em T. claussenii, $R$. purshiana, C. sylvestris e T. micrantha, revelaram um maior potencial de dispersão de suas sementes em comparação às demais espécies, o que decorreu, principalmente, da abundância dos frutos nas plantas (obs. pessoal) e ao seu pequeno tamanho (Tab. 2), facilitando o consumo sem a necessidade de mandibulação para uma grande variedade de aves, especialmente aquelas de menor porte e com bicos mais estreitos, tais como Dacnis cayana e Nemosia pileata, com respectivamente $13,3 \mathrm{~g}$ e $14 \mathrm{~g}$ de massa corporal e $7,1 \mathrm{~mm}$ e $7,5 \mathrm{~mm}$ de largura máxima do bico, segundo Argel-de-Oliveira (1999). As espécies T. claussenii e C. sylvestris, merecem maior destaque, já que ambas são atrativas para um número significativo de espécies, o que deve estar relacionado à alta proporção de polpa em seus frutos (Tab. 3) e à presença de arilo em suas sementes. $\mathrm{O}$ teor de proteínas e lipídios de um fruto é indicador do seu valor nutricional, o qual pode influenciar a preferência das aves (Martin, 1985). Neste sentido, destacam-se as espécies cujos frutos, além de pequenos, apresentam sementes ariladas, pois estão entre as mais nutritivas devido ao alto teor de lipídios geralmente presente no arilo (Howe \& Estabrook 1977; Pizo 1997). Portanto, C. sylvestris e T. claussenii possuem características bastante favoráveis à manutenção das aves frugívoras (facultativas ou não) e, consequentemente, à sustentabilidade da própria comunidade vegetal, apresentando grande potencial de utilização em projetos de recuperação e restauração florestal. 
Tabela 4. Matriz de interação entre as espécies de aves e de plantas da área de estudo, indicando o número de frutos consumidos durante as sessões sistemáticas de observação focal. É assinalado também o consumo ad libitum, indicado por "x".

\begin{tabular}{|c|c|c|c|c|c|c|c|c|c|c|c|c|c|c|c|c|c|}
\hline \multirow{2}{*}{ Espécies de aves } & \multirow{2}{*}{ Nomes populares } & \multirow{2}{*}{ Dieta } & \multicolumn{13}{|c|}{ Espécies vegetais $^{3}$} & \multirow{2}{*}{ Total } & \multirow{2}{*}{$\mathbf{P C}^{4}$} \\
\hline & & & ST & $S R$ & MA & TC & $F C$ & $E J$ & $M C$ & $R P$ & CS & $A A$ & $S G$ & $T M$ & CSp. & & \\
\hline \multicolumn{18}{|l|}{ Columbidae } \\
\hline Patagioenas picazuro (Temminck, 1813) & pombão & Frugívoro & & & 1 & & & & & & & & & & & 1 & 0,06 \\
\hline \multicolumn{18}{|l|}{ Psittacidae } \\
\hline Forpus xanthopterygius (Spix 1824) & tuim & Onívoro & & & 1 & & $\mathrm{x}$ & & & & 22 & 2 & & 60 & 10 & 95 & 6,04 \\
\hline Brotogeris chiriri (Vieillot, 1818) & $\begin{array}{l}\text { periquito-de- } \\
\text { encontro-amarelo }\end{array}$ & Frugívoro & & & & & & & & & & & & & & - & - \\
\hline Pionus maximiliani (Kuhl, 1820) & maitaca-verde & Frugívoro & & & 1 & & & & & & & & & & & 1 & 0,06 \\
\hline \multicolumn{18}{|l|}{ Tyrannidae } \\
\hline $\begin{array}{l}\text { Camptostoma obsoletum } \\
\text { (Temminck, 1824) }\end{array}$ & risadinha & Insetívoro & $\mathrm{x}$ & & & & & & & & & & & & & - & - \\
\hline $\begin{array}{l}\text { Tolmomyias sulphurescens (Spix, } \\
\text { 1825) }\end{array}$ & bico-chato-de-orelha-preta & Insetívoro & & & & & & & & & 6 & & & 2 & & 8 & 0,51 \\
\hline Elaenia spectabilis Pelzeln, 1868 & guaracava-grande & Onívoro & & & & & & & & & & 1 & & & & 1 & 0,06 \\
\hline Elaenia flavogaster (Thunberg, 1822) & chibum & Onívoro & & & 2 & 5 & & & & & 9 & 1 & & & & 17 & 1,08 \\
\hline Elaenia sp. & - & Onívoro & $\mathrm{x}$ & & 4 & 1 & & & & & 14 & 11 & & & & 30 & 1,91 \\
\hline Satrapa icterophrys (Vieillot, 1818) & suiriri-pequeno & Insetívoro & & & 1 & & & & & & & & & & & 1 & 0,06 \\
\hline Pitangus sulphuratus (Linnaeus, 1766) & bem-te-vi & Onívoro & & & 8 & 2 & & & $\mathrm{x}$ & & & & & & & 10 & 0,63 \\
\hline $\begin{array}{l}\text { Myiodynastes maculatus } \\
\text { (Statius Muller, 1776) }\end{array}$ & bem-te-vi-rajado & Onívoro & & & & 20 & 1 & & & & 1 & & & & & 22 & 1,40 \\
\hline Myiozetetes similis (Spix, 1825) & $\begin{array}{l}\text { bentevizinho-de- } \\
\text { penacho-vermelho }\end{array}$ & Onívoro & & & 12 & 27 & & & & & 4 & 1 & & 20 & & 64 & 4,07 \\
\hline Empidonomus varius (Vieillot, 1818) & peitica & Onívoro & & & $\mathrm{x}$ & 5 & & & & & & & & & & 5 & 0,32 \\
\hline Myiarchus ferox (Gmelin, 1789) & maria-cavaleira & Insetívoro & & & & 12 & & & & & 14 & & & & & 26 & 1,65 \\
\hline $\begin{array}{l}\text { Tyrannus melancholicus } \\
\text { Vieillot, } 1819\end{array}$ & suiriri & Insetívoro & & & & 1 & & & & & & 1 & & & & 2 & 0,13 \\
\hline \multicolumn{18}{|l|}{ Vireonidae } \\
\hline Cyclarhis gujanensis (Gmelin, 1789) & pitiguari & Insetívoro & $\mathrm{x}$ & & & & & 1 & & & 12 & & & & & 13 & 0,83 \\
\hline Vireo olivaceus (Linnaeus, 1766) & juruviara & Onívoro & & & & 19 & & & & & 1 & & & & & 20 & 1,27 \\
\hline \multicolumn{18}{|l|}{ Turdidae } \\
\hline Turdus amaurochalinus Cabanis, 1850 & sabiá-poca & Onívoro & $\mathrm{x}$ & & 31 & 1 & & 3 & & & & 1 & & & & 36 & 2,29 \\
\hline Turdus leucomelas Vieillot, 1818 & sabiá-barranco & Onívoro & & & 29 & 12 & & $\mathrm{x}$ & 2 & & 1 & & & & & 44 & 2,79 \\
\hline Turdus ruvifentris Vieillot, 1818 & sabiá-laranjeira & Onívoro & & & 8 & & & & & & & & & & & 8 & 0,51 \\
\hline \multicolumn{18}{|l|}{ Coerebidae } \\
\hline Coereba flaveola (Linnaeus, 1758) & cambacica & Onívoro & $\mathrm{x}$ & 7 & & 1 & 1 & 4 & 30 & & & 7 & & & 2 & 52 & 3,30 \\
\hline \multicolumn{18}{|l|}{ Thraupidae } \\
\hline Thraupis palmarum (Wied, 1823) & sanhaço-do-coqueiro & Onívoro & $\mathrm{x}$ & & & & & & & & & & & & 1 & 1 & 0,06 \\
\hline Thraupis sayaca (Linnaeus, 1766) & sanhaçu-cinzento & Onívoro & $\mathrm{x}$ & & 253 & 7 & $\mathrm{x}$ & 14 & 38 & 104 & 17 & 11 & 6 & 1 & 5 & 456 & 28,97 \\
\hline $\begin{array}{l}\text { Thlypopsis sordida } \\
\text { (d'Orbigny \& Lafresnaye, 1837) }\end{array}$ & saí-canário & Onívoro & $\mathrm{x}$ & & 1 & 2 & & & & & 2 & 1 & & & & 6 & 0,38 \\
\hline Tangara cayana (Linnaeus, 1766) & saíra-amarela & Onívoro & 2 & & 51 & 3 & & & 2 & 37 & 17 & 19 & $\mathrm{x}$ & 39 & 4 & 174 & 11,05 \\
\hline Nemosia pileata (Boddaert, 1783) & saíra-de-chapéu-preto & Onívoro & & & & & & 14 & 6 & & 41 & & & & & 61 & 3,87 \\
\hline $\begin{array}{l}\text { Conirostrum speciosum } \\
\text { (Temminck, 1824) }\end{array}$ & $\begin{array}{l}\text { figuinha-de-rabo- } \\
\text { castanho }\end{array}$ & Insetívoro & & & $\mathrm{x}$ & & & $\mathrm{x}$ & & & & 1 & & & & 1 & 0,06 \\
\hline Ramphocelus carbo (Pallas, 1764) & pipira-vermelha & Onívoro & & & 6 & 28 & & & 7 & 18 & 32 & 20 & $\mathrm{x}$ & & 1 & 112 & 7,12 \\
\hline Tachyphonus coronatus (Vieillot, 1822) & tiê-preto & Onívoro & & & & 3 & & 1 & 16 & 4 & 8 & 18 & & & & 50 & 3,18 \\
\hline Dacnis cayana (Linnaeus, 1766 ) & saí-azul & Onívoro & $\mathrm{x}$ & & & 75 & & & 1 & & 5 & 1 & & 166 & & 248 & 15,76 \\
\hline \multicolumn{18}{|l|}{ Emberezidae } \\
\hline $\begin{array}{l}\text { Zonotrichia capensis } \\
\text { (Statius Muller, 1776) }\end{array}$ & tico-tico & Granívoro & & & & & & & $\mathrm{x}$ & & & & & & & - & - \\
\hline Cardinalidae & & & & & & & & & & & & & & & & & \\
\hline Habia rubica (Vieillot, 1817) & tiê-do-mato-grosso & Onívoro & & & & & & & & & $\mathrm{x}$ & & & & & - & - \\
\hline Parulidade & & & & & & & & & & & & & & & & & \\
\hline Parula pitiayumi (Vieillot, 1817) & mariquita & Insetívoro & & & $\mathrm{x}$ & & & & & & & & & & & - & - \\
\hline Icteridae & & & & & & & & & & & & & & & & & \\
\hline Icterus cayanensis (Linnaeus, 1766) & encontro & Onívoro & & & 2 & & & & 4 & & & & & & & 6 & 0,38 \\
\hline Fringillidae & & & & & & & & & & & & & & & & & \\
\hline Euphonia chlorotica (Linnaeus, 1766) & fim-fim & Onívoro & & & 3 & & & & & & & & & & & 3 & 0,19 \\
\hline $\begin{array}{l}\text { Euphonia cyanocephala } \\
\text { (Vieillot, 1818) }\end{array}$ & gaturamo-rei & Onívoro & & & $\mathrm{x}$ & & & & & & & & & & & - & - \\
\hline Euphonia violacea (Linnaeus, 1758) & gaturamo-verdadeiro & Onívoro & & $\mathrm{x}$ & & & & & & & & & & & & - & - \\
\hline
\end{tabular}




\section{Avifauna consumidora}

A avifauna consumidora de frutos se mostrou bastante generalista, sendo representada principalmente por espécies onívoras, de pequeno tamanho corporal e hábitos oportunistas, o que reflete a própria composição da comunidade de aves do reflorestamento e da matriz circundante (obs. pessoal), onde predominam espécies onívoras e insetívoras menos especializadas, típicas de ambientes alterados, de acordo com Willis (1979).

Quanto ao consumo, não houve diagnóstico explícito de seleção dos frutos no tocante às dimensões, afirmação corroborada pela elevada ocorrência de consumo de partes de polpa: frequentemente aves com porte e largura máxima de bico reduzidos também consumiram frutos de tamanho médio, os quais elas seriam incapazes de ingerir inteiros. É possível citar como exemplos Euphonia chlorotica e T. cayana - que possuem, respectivamente, $6,7 \mathrm{~mm}$ e 7,8 mm de largura máxima de bico (Argel-de-Oliveira 1999) consumindo porções da polpa dos frutos de M. azedarach. Tal situação está relacionada às condições impostas por ambientes alterados e com maiores limitações de recursos, explorados geralmente por espécies de aves oportunistas e que, portanto, não apresentam a seletividade dos frugívoros especialistas de áreas florestais maduras e conservadas, onde existem padrões co-evolutivos de forrageio (Jordano et al. 2006).

Os resultados evidenciaram que as aves das famílias Turdidae e Tyrannidae apresentam grande potencial como dispersoras, pois geralmente se alimentam dos frutos sem destruir as sementes, assim como observado por Howe \& Estabrook (1977). As espécies Forpus xanthopterygius e Pionus maximiliani atuaram como potenciais predadoras das sementes, já que quebraram e maceraram os frutos antes de ingeri-los, o que é comum na família Psittacidae, de acordo com Pizo (1997). Nos demais casos, aparentemente a movimentação da mandíbula ocorreu somente para o ajuste do fruto no bico, como forma de facilitar a ingestão (Moermond 1983).

A significativa riqueza de aves consumindo frutos e a presença de espécies potencialmente dispersoras evidenciaram a relevância da interação frugívoro-planta para florestas em regeneração/recuperação, assim como para a avifauna, especialmente em épocas de escassez de alimentos.

\section{Considerações finais}

Os principais consumidores e potenciais dispersores nos ecossistemas estudados são espécies de aves onívoras e oportunistas, comuns inclusive em áreas antrópicas; e as espécies vegetais são frequentes em estágios iniciais de sucessão ecológica e ecossistemas perturbados. No entanto, segundo Francisco \& Galetti (2001), a atração de aves generalistas pode aumentar as chances das espécies vegetais se estabelecerem em ecossistemas degradados, como em pastagens abandonadas, parques urbanos e fragmentos florestais depauperados. Portanto, espécies ornitocóricas mais ruderais são de grande importância para a recolonização florística e faunística em ambientes alterados.

É possível concluir que o reflorestamento misto, embora apresente espécies exóticas problemáticas como $M$. azedarach, está sendo ecologicamente mais funcional para a avifauna em detrimento do fragmento florestal; devido à proximidade entre eles, este último pode estar sendo favorecido em termos de manutenção da comunidade de aves residentes e visitantes pelos recursos alimentares oferecidos na área de recomposição florestal.

\section{Agradecimentos}

À Fundação Patrimônio Histórico da Energia e Saneamento, por permitir a realização do estudo no Museu da Energia Usina-Parque do Corumbataí; ao Dagmar Carnier Neto, por auxiliar no trabalho de campo; ao Programa de Pós-Graduação em Ecologia e Recursos Naturais da Universidade Federal de São Carlos e ao CNPq (Conselho Nacional de Desenvolvimento Científico e Tecnológico), pela bolsa de Mestrado concedida à primeira autora (Processo: 133971/2007-4).

\section{Referências bibliográficas}

Argel-de-Oliveira, M.M. 1999. Frugivoria por aves em um fragmento de floresta de restinga no Estado do Espírito Santo, Brasil. Tese de Doutorado. UNICAMP, Campinas.

Cáceres, N.C. \& Moura, M.O. 2003. Fruit removal of a wild tomato, Solanum granulosoleprosum Dunal (Solanaceae), by birds, bats and non-flying mammals in an urban Brazilian environment. Revista Brasileira de Zoologia 20(3): 519-522.

Castro, E.R. \& Galetti, M. 2004. Frugivoria e dispersão de sementes pelo lagarto teiú Tupinambis merianae (Reptilia: Teiidae). Papéis Avulsos de Zoologia 44(6): 91-97.

CBRO (Comitê Brasileiro De Registros Ornitológicos) 2008. Lista das aves do Brasil. Disponível em www.cbro.org.br (Acesso em 4/05/2010).

CESP (Companhia Energética de São Paulo) 1982. Corumbataí: restauração da usina hidrelétrica. São Paulo, CESP.

Durigan, G.; Franco, G.A.D.; Saito, M \& Baitello, J.B. 2000. Estrutura e diversidade do componente arbóreo da floresta na Estação Ecológica dos Caetetus, Gália, SP. Revista Brasileira de Botânica 23: 371-383.

Fleming, T.H.; Breitwisch, R. \& Whitesides, G.H. 1987. Patterns of tropical vertebrate diversity. Annual Review of Ecology and Systematics 18: 91-109.

Francisco, M.R. \& Galetti, M. 2001. Frugivoria e dispersão de sementes de Rapanea lancifolia (Myrsinaceae) por aves numa área de cerrado do Estado de São Paulo, sudeste do Brasil. Ararajuba 9(1): 13-19.

Foster, M.S. 1977. Ecological and nutritional effects of food scarcity on a tropical frugivorous bird and its fruit source. Ecology 58: 73-85.

Gosper, C.R.; Stansbury, C.D. \& Vivian-Smith, G. 2005. Seed dispersal of fleshy-fruited invasive plants by birds: contributing factors and management options. Diversity Distributions 11: 549-558.

Howe, H.F. \& Estabrook, G.F. 1977. On intra-especific competition for avian dispersers in tropical trees. American Naturalist 111: 817-832.

Howe, H.F. 1984. Implications of seed dispersal by animals for tropical reserve management. Biological Conservation 30: 261-281.

Jordano, P.M.; Galetti, M; Pizo, M.A. \& Silva, W.R. 2006. Ligando frugivoria e dispersão de sementes à biologia da conservação. Pp. 411-436. In: 
Duarte, C.F.; Bergallo, H.G.; Alves, M.A.S. \& Van Sluys, M. (Eds.). Biologia da conservação: essências. São Paulo, Editorial Rima.

Krügel, M.M.; Burger, M.I. \& Alves, M.A.S. 2006. Frugivoria por aves em Nectandra megapotamica (Lauraceae) em uma área de Floresta Estacional Semidecidual no Rio Grande do Sul, Brasil. Iheringia (96) 1: 17-24

Lorenzi, H. 1992. Árvores brasileiras. Nova Odessa, Editora Plantarum.

Martin, T.E. 1985. Resource selection by tropical frugivorous birds: integrating multiple interactions. Oecologia 66: 563-573.

Moermond, T.C. 1983. Suction-drinking in tanagers and its relation to fruit handling. Ibis 125: 545-549.

Molinari, J. 1993. El mutualismo entre frugivoros y plantas en las selvas tropicales: aspectos paleobiologicos, autoecologias, papel comunitário. Acta Biologica Venezuelica 14(4): 1-44.

Morellato, L.P.C. 1991. Estudo da fenologia de árvores, arbustos e lianas de uma floresta semidecídua no sudeste do Brasil. Tese de Doutorado, Campinas, UNICAMP.

Pijl, L.V.D. 1969. Principles of dispersal in higher plants. Berlin, SpringerVerlag.

Pizo, M.A. 1997. Seed dispersal and predation in two populations of Cabralea canjerana (Meliaceae) in the Atlantic forest of southeastern Brazil. Journal of Tropical Ecology 13: 559-578.

Reis, A.; Zambonin, R.M. \& Nakazono, E.M. 1999. Recuperação de áreas florestais degradadas utilizando a sucessão e as interações planta- animal. Série Recuperação, Caderno 14, São Paulo, Conselho Nacional da Reserva da Biosfera da Mata Atlântica.

Rodrigues, R.R. \& Gandolfi, S. (2000) Conceitos, tendências e ações para a recuperação de florestas ciliares. Pp.235-247. In: Rodrigues, R.R. \& Leitão-Filho, H.F. (Eds). Matas ciliares: conservação e recuperação. São Paulo, EDUSP/FAPESP.

Sick, H. 1997. Ornitologia brasileira. Rio de Janeiro, Editora Nova Fronteira.

Silva, W.R. 2003. A importância das interações planta-animal nos processos de Restauração. Pp.77-90. In: Kageyama, P.Y.; Oliveira, R.E.; Moraes, L.F.D.; Engel, V.L. \& Gandara, F.B. (Eds.). Restauração ecológica de ecossistemas naturais. Botucatu, Fundação de Estudos e Pesquisas Agrícolas e Florestais.

Stiles, F.G. 1976. Taste preferences, color preferences, and flower choice in hummingbirds. Condor 78: 10-26.

Troppmair, H. 1992. Atlas da qualidade ambiental e de vida de Rio Claro - SP. Arquivo público e histórico do município de Rio Claro. Rio Claro, Ultra Copy.

Willis, E.O. 1979. The composition of avian communities in remanescent woodlots in southern Brazil. Papéis Avulsos de Zoologia 33(1): 1-25.

Wunderle, J.M. 1997. The role of animal seed dispersal in accelerating native forest regeneration on degraded tropical lands. Forest Ecology and Management 99: 223-235. 